\title{
ACTIVIDAD ANTIOXIDANTE DE METABOLITOS DE FLAVONOIDES ORIGINADOS POR LA MICROFLORA DEL INTESTINO HUMANO
}

\author{
Felipe Surco-Laos $^{1 *}$, Manuel Valle Campos ${ }^{1}$, Eddie Loyola $^{1}$, Montserrat Dueñas $^{2}$, \\ Celestino Santos ${ }^{2}$.
}

\begin{abstract}
RESUMEN
Los flavonoides y demás compuestos fenólicos cuando son ingeridos son metabolizados hasta ácidos fenólicos, que se clasifican en benzoicos, fenilacéticos y cinámicos, principalmente. Mediante métodos "in vitro" (ABTS y FRAP) se determinó la capacidad antioxidante de los ácidos fenólicos de interés metabólico potencial. Los valores obtenidos varían según la reacción química implicada y la estructura de los compuestos, en la cual influye el número de radicales, su posición en el anillo bencénico con relación al grupo carboxílico y la longitud de la cadena de este último. En las interacciones entre las mezclas de ácidos se observa principalmente un efecto antagónico, no existiendo ningún patrón estructural que lo explique.
\end{abstract}

Palabras clave: Actividad antioxidante, ácidos fenólicos, ABTS, FRAP

\section{ANTIOXIDANTS ACTIVITY OF FLAVONOID METABOLITES CAUSED BY THE HUMAN GUT MICROFLORA}

\begin{abstract}
Flavonoids and more phenolic compounds when ingested are metabolized to phenolic acids, which are classified as benzoic, phenylacetic and cinnamic primarily, by "in vitro" methods (ABTS and FRAP), the antioxidant capacity of phenolic acids of potential metabolic interest was determined. The values vary by chemical reaction involved and the structure of the compounds, which influences the number of radicals, their position on the benzene ring relative to the carboxylic group and the chain length of the latter. In interactions between mixtures of acids a mainly antagonistic effect is observed, there is no structural pattern that explains it.
\end{abstract}

Key words: Antioxidants activity, acids phenolic, ABTS, FRAP.

\footnotetext{
${ }^{1}$ Facultad de Farmacia y Bioquímica de la Universidad San Luis Gonzaga de Ica. *felipesurco@gmail.com

${ }^{2}$ Grupo de Investigación de Polifenoles. Universidad de Salamanca. España
} 


\section{INTRODUCCIÓN}

La importancia de la oxidación de los alimentos en el cuerpo está ampliamente documentada y muchos compuestos de reconocidas actividad antioxidante como: rutina, isoquercetina, quercetina, flavonoides, hidroxicinamatos, entre otros, actúan como protectores del efecto de las especies reactivas de oxígeno y otros radicales libres; lo que les da un rol de protección en la reducción del riesgo de enfermedades coronarias, enfermedades degenerativas y ciertos tipos de cánceres ${ }^{1-3}$. La clave crucial en la prevención de las enfermedades es la acción que ejercen después de ser consumidos ${ }^{4}$.

Dos de los sitios principales del metabolismo de los polifenoles es el hígado y la flora del colon. Existe evidencia que la o-metilación, sulfatación y glucuronidación de los grupos hidroxilos es en el hígado. La fisión bacterial del anillo de flavonoides ocurre en el colon 5 . Los polifenoles no son completamente absorbidos como tales desde el tracto gastrointestinal, sino que sufren los procesos de metabolización o degradación por la presencia de muchas enzimas y una variada microflora en todo el tracto gastrointestinal convirtiéndolos a diferentes metabolitos entre los que predominan los ácidos hidroxibenzoicos, hidroxifenilacéticos, hidroxifenilpropionicos, entre otros ${ }^{2,6-8}$. Ellos y sus metabolitos acumulados pueden ejercer su efecto fisiológico.

Se ha demostrado ${ }^{1,9}$ que algunos flanovoides y derivados son desconjugados e hidroxilados in vitro por la flora colónica, produciéndose como principal metabolito el ácido 3,4-dihidroxifenilacético, el cual fue dehidroxilado a 3-hidroxifenilacético, 3-fenilpropiónico, etc.

El objetivo de este estudio fue investigar la actividad antioxidante y su relación con respecto a la estructura de los ácidos fenólicos que resultan como metabolitos, originados por la microflora del intestino humano a partir de muchos compuestos polifenolicos, empleando dos métodos que poseen mecanismos diferentes: FRAP (capacidad para reducir el $\mathrm{Fe}^{3+} \mathrm{a} \mathrm{Fe}^{2+}$ ) y ABTS (capacidad para donar hidrógeno o electrones); asimismo, las interacciones entre ácidos fenólicos en sistemas binarios fueron determinadas simultáneamente en proporciones equimolar, para estudiar los efectos sinérgicos o antagónicos que ocurren entre pares de ácidos en una mezcla.

\section{PARTE EXPERIMENTAL}

\section{Reactivos}

- Ácidos fenólicos: Ácido 4-hidroxi-benzoico.- Fluka, ácido gálico (3,4,5-trihidroxibenzoico).- Merck, ácido vaníllico (4-hidroxi-3-metoxibenzoico), ácido siríngico (4-hidroxi-3,5-dimetoxi-benzoico), ácido floroglucinol (1,3,5-trihidroxibenceno), ácido 3-(4-hidroxifenil) propiónico, ácido 2,4-dihidroxibenzoico, ácido protocatéquico (3,4-dihidroxibenzoico), ácido p-cumárico (3-(4-hidroxifenil) cinámico), ácido 3,4-dihidroxifenilacético, ácido transferúlico, 
ácido homovaníllico 4-hidroxi-3-metoxifenilacético, ácido 3-hidroxifenilacético, ácido 3-fenilpropiónico, ácido 4-hidroxifenilacético, ácido caféico (3,4-dihidroxifenilcinámico).- Sigma-Aldrich, todos de una pureza mayor al 98\%.

- Trolox ((+/-)-6-hidroxi-2,5.7,8-tetrametilcroman-2-carboxilic ácido).- Fluka

- $\quad$ TPTZ (2,4,6-tripiridil-s-triazina). Sigma

- $\quad$ ABTS (2,2'-azino-bis (3-etilbenzotiazolin-6-sulfónico). Fluka

\section{Métodos}

\section{- Método del FRAP}

Se siguió el procedimiento descrito por Benzie y Strain (1999) ${ }^{10}$ con ligeras modificaciones. Para iniciar el análisis se preparó el reactivo de trabajo, que consiste en una mezcla de tampón acetato $300 \mathrm{mM}(\mathrm{pH}=3,6)$, TPTZ $10 \mathrm{mM}$ en $\mathrm{HCl} 40 \mathrm{mM}$ y tricloruro férrico $\left(\mathrm{FeCl}_{3} .6 \mathrm{H}_{2} \mathrm{O}\right) 20 \mathrm{mM}$ en una proporción 10:1:1 (v:v:v). Una vez preparado, se añadió $3 \mathrm{~mL}$ de este reactivo en una cubeta, y se midió la absorbancia a $593 \mathrm{~nm}$. Posteriormente, se agregó $100 \mu \mathrm{L}$ de una de las soluciones de los ácidos $(0,25$ $-1,0 \mathrm{mM})$ y se agitó en un vórtex durante 30 segundos. Después de 6 minutos de incubación a temperatura ambiente, se realizó la lectura de absorbancia nuevamente a $593 \mathrm{~nm}$, al que se restó el valor de la absorbancia inicial. Las muestras se ensayaron por triplicado.

- Método reacción con el radical 2,2'-azino-bis-(3-etilbenztiazolín-6-sulfonato de amonio) (ABTS):

Se realizó según el método propuesto, empleando la capacidad antioxidante del ABTS* y su habilidad de secuestrar radicales de larga vida.

En este método, la generación del radical ABTS* se produce por medio químico mediante la adición de persulfato de potasio antes de la adición de la muestra, lo que evita que los componentes de la misma pueda reaccionar con el reactivo; otra ventaja es que se trabaja a $\mathrm{pH}$ fisiológico $(\mathrm{pH}=7,1)$, a una temperatura de $37^{\circ} \mathrm{C}$ simulando las condiciones fisiológicas.

Se pesan 0,0504 g de la sal amónica cristalizada de ABTS y se disuelve en $5 \mathrm{~mL}$ de agua ultra pura, luego se adiciona $6,7 \mathrm{mg}$ de persulfato de potasio $\left(\mathrm{K}_{2} \mathrm{~S}_{2} \mathrm{O}_{8}\right)$ y se deja agitando por espacio de media hora envuelta en papel aluminio protegido de la luz, pasado este tiempo se transfiere a un matraz volumétrico de $10 \mathrm{~mL}$ y se enrasa con agua ultra pura y se deja reaccionar a temperatura ambiente y protegido de la luz durante 12 a 18 horas. Transcurrido el tiempo correspondiente se toma una alícuota de $1 \mathrm{ml}$ y se adiciona aproximadamente $70 \mathrm{~mL}$ de tampón fosfato de $\mathrm{pH}$ 7,1 y se mide la absorbancia a $734 \mathrm{~nm}$ el cual debe estar entre 0,680 $\pm 0,02$.

Para la medida de la actividad antioxidante se tomaron $1 \mathrm{~mL}$ del radical ABTS* en una cubeta y se midió su absorbancia inicial a $734 \mathrm{~nm}$ con el equipo termostatizado a $37{ }^{\circ} \mathrm{C}$, posteriormente se añadieron $25 \mu \mathrm{L}$ de una de las soluciones de los ácidos respectivos (el cual debe haber estado en un baño maría a $37^{\circ} \mathrm{C}$ ), se mezcla durante 10 
segundos en un vortex, después de 4 minutos de incubación se midió la absorbancia final a $734 \mathrm{~nm}$. Todas las muestras se analizaron por triplicado.

Al igual que en el método de FRAP, los resultados se expresaron como la concentración $1 \mathrm{mM}$ de ácido que tiene la misma actividad antioxidante equivalente de $\mathrm{mM}$ de Trolox correspondiente. Para ello es necesario realizar una curva de calibración con diferentes concentraciones de Trolox frente a las correspondientes variaciones de absorbancias del radical.

En los casos en los cuales soluciones $1 \mathrm{mM}$ de ácidos se obtuvo como resultado no detectable se probó, hasta concentraciones de $10 \mathrm{mM}$.

\section{RESULTADOS Y DISCUSIÓN}

La actividad antioxidante de los ácidos fenólicos y sus ésteres depende tanto del número de grupos hidroxilos en la molécula, la posición de estos en la estructura del anillo bencénico en relación al grupo carboxílico y la extensión de la estructura del grupo carboxílico.

No hay un único método que defina totalmente la eficacia antioxidante de una sustancia o mezcla compleja, por lo cual se recomienda el uso de más de un método para determinar la capacidad antioxidante de una muestra.

En este trabajo se determinó la actividad antioxidante de los ácidos fenólicos por 2 métodos diferentes que implican 2 mecanismos distintos.

Tabla 1. Coordenadas del área de trabajo

\begin{tabular}{|c|c|c|}
\hline Ac. Hidroxibenzoicos & ABTS (mM) & FRAP (mM) \\
\hline Ac. 2-hidroxibenzoico & $0,04 \pm 0,01$ & N.D. \\
\hline Ac. 4-hidroxibenzoico & $0,04 \pm 0,01$ & N.D. \\
\hline $\begin{array}{l}\text { Ac. 3,4-dihidroxibenzoico } \\
\text { (protocatéquico) }\end{array}$ & $1,04 \pm 0,02$ & $1,03 \pm 0,05$ \\
\hline Ac. 2,4-dihidrobenzoico & $1,93 \pm 0,04$ & $0,02 \pm 0,01$ \\
\hline $\begin{array}{l}\text { Ac. 4-hidroxi-3-metoxibenzoico } \\
\text { (vaníllico) }\end{array}$ & $1,52 \pm 0,02$ & $0,29 \pm 0,01$ \\
\hline Ac. 3,4,5-trihidroxibenzoico (gálico) & $3,90 \pm 0,04$ & $2,59 \pm 0,04$ \\
\hline $\begin{array}{l}\text { Ac. 3,5-dimetoxi-4-hidroxibenzoico } \\
\text { (siríngico) }\end{array}$ & $1,61 \pm 0,01$ & $1,79 \pm 0,06$ \\
\hline
\end{tabular}

N.D. $=$ no detectable 
En términos de capacidad de donación de hidrogeno, el ácido monohidroxibenzoico en posición orto (2-hidroxibenzoico) y para (4-hidroxibenzoico) no muestra actividad antioxidante $(0,04)$ contra radicales generados en fases acuosa (ABTS*), coincidiendo con la revisión hecha por Rice-Evans ${ }^{8}$ que indica que solo el monohidroxibenzoico en posición meta presenta actividad antioxidante $(0,84)$.

Los ácidos dihidroxibenzoicos muestran una respuesta antioxidante dependiente de las posiciones relativas de los grupos hidroxil en el anillo. Conjugando con los datos publicados podemos establecer un orden de relación entre la dihidroxilación y las posiciones en el anillo aromático: así la proximidad del grupo carboxilo a los sustituyentes difenólicos aparentemente influencia la disponibilidad de los $\mathrm{H}$ con la posición y permite establecer un orden creciente de efectividad: orto/meta 2,5 (1,04); meta/para 3,4 $(1,19)$; orto/meta 2,3 $(1,46)$; orto/para 2,4 $(1,93)$; meta/meta, 3,5 $(2,15)$. Cuando en la posición 3, un grupo hidroxilo es sustituido por un metoxilo se observa un incremento de la actividad antioxidante: ácido protocatéquico $(1,04)$, ácido vaníllico $(1,52)$; mientras que en los trisustituidos como el ácido gálico $(3,9)$, la presencia de los 2 grupos metoxi adyacentes a el grupo $\mathrm{OH}$ en posición p-hidroxibenzoico acentúa la reducción de disponibilidad de $\mathrm{H}$ y por tanto reduce la actividad antioxidante, el caso del ácido siríngico $(1,62)$.

Por el método FRAP podemos observar que los ácidos monohidroxil benzoico no presentan actividad, sin importar la posición del $\mathrm{OH}$ en la estructura del anillo; de los dihidroxisustituidos sólo el 3,4-dihidroxibenzoico muestra actividad (1,79), mientras que la sustitución del $\mathrm{OH}$ por un grupo $-\mathrm{OCH}_{3}$ en la posición 3 reduce su actividad antioxidante a un valor mínimo $(0,29)$ y en el caso de los trihidroxi sustituidos la actividad se incrementa considerablemente, como el ácido gálico $(2,59)$; pero al sustituirse los grupos $\mathrm{OH}$ adyacentes al $\mathrm{OH}$ de posición para, la actividad también decrece, ácido siríngico $(1,79)$ mostrando el mismo comportamiento que los dihidroxisustituidos (tabla 1).

Tabla 2. Actividad antioxidante (TEAC) de ácidos fenilacéticos

\begin{tabular}{cccc}
\hline Ac. Fenil Acético & ABTS (mM) & FRAP (mM) \\
\hline Ac. 2-hidroxifenilacético & 0,99 & $\pm 0,09$ & $0,04 \pm 0,00$ \\
Ac. 3-hidroxifenilacético & 0,95 & $\pm 0,04$ & N.D. \\
Ac. 4-hidroxifenilacético & 1,13 & $\pm 0,09$ & $0,05 \pm 0,00$ \\
Ac. 3,4-dihidroxifenilacético & 3,50 & $\pm 0,02$ & $4,03 \pm 0,1$ \\
$\quad \begin{array}{c}\text { Ac. 4-hidroxi-3- } \\
\text { metoxifenilacético }\end{array}$ & 1,77 & $\pm 0,04$ & $1,88 \pm 0,01$ \\
\hline
\end{tabular}

N.D. $=$ no detectable 
Los ácidos fenilacéticos, en los cuales se ha introducido un grupo $-\mathrm{CH}_{2}$ - entre el anillo fenólico y el grupo carboxílico, muestran la siguiente actividad antioxidante: los derivados orto, meta y para tienen actividad cercana a la unidad, o-hidroxifenilacético $(0,99)$, $m$-hidroxifenilacético $(0,95)$ y $p$-hidroxifenilacético $(1,13)$; este último tiene una diferencia con el declarado por Rice-Evans ${ }^{8}$, que reporta una actividad de 0,34 ; los dihidroxisustituidos presentan una mayor actividad que sus homólogos hidroxibenzoicos, observándose que en este caso la sustitución del grupo $\mathrm{OH}$ en posición meta por el grupo $-\mathrm{OCH}_{3}$ conduce a una disminución de la actividad: 3,4-dihidroxifenilacético $(3,50)$ y el 4-OH, 3-OCH -fenilacético $(1,77)^{13}$.

En el método FRAP ocurre lo mismo que con sus homólogos hidroxil benzoicos; así el 3,4-dihidroxifenilacético $(4,03), 4-\mathrm{OH}, 3-\mathrm{OCH}_{3}$-fenilacético $(1,88)$, al sustituir un $\mathrm{OH}$ por un metoxi disminuye con capacidad antioxidante (tabla 2).

Tabla 3. Actividad antioxidante (TEAC) de ácidos hidroxicinámicos

\begin{tabular}{ccc}
\hline Ac. Hidróxicinámico & ABTS $^{*}(\mathbf{m M})$ & FRAP $(\mathbf{m M})$ \\
\hline Ac. o-cumárico $^{\mathrm{a}}$ & $0,99 \pm 0,15$ & $0,04 \pm 0,00$ \\
Ac. p-cumárico & $2,43 \pm 0,04$ & $0,09 \pm 0,00$ \\
Ac. ferúlico & $2,38 \pm 0,01$ & $1,08 \pm 0,05$ \\
Ac. caféico & $1,23 \pm 0,03$ & $1,23 \pm 0,07$ \\
\hline
\end{tabular}

${ }^{\mathrm{a}}$ Tomado de Rice-Evans ${ }^{8}$

Ácidos hidrocinámicos por ABTS*. Se observa que una mayor hidroxilación tiene un efecto negativo en la actividad antioxidante: ácido caféico $(1,23)$, ácido ferúlico $(2,38), p$-cumárico $(2,48)$, con la excepción de $o$-cumárico en el que dicha posición disminuye su actividad, a pesar de mostrar valores ligeramente superior a reportes anteriores ${ }^{2,8}$, presentan la misma tendencia; sin embargo, se muestran superior que sus homólogos fenilacético; mientras que por FRAP los resultados de actividad antioxidante se incrementan con la hidroxilación y coinciden con los estudios que demuestran su efectividad sobre el periodo inducción de autoxidación de las grasas: ácidos caféico $>$ ferúlico $>$ p-cumárico ${ }^{13}$.

Tabla 4. Actividad antioxidante (TEAC) de otros ácidos

\begin{tabular}{ccc}
\hline Otros & ABTS (mM) & FRAP (mM) \\
\hline Ac. floroglucinol & $2,51 \pm 0,02$ & N.D. \\
Ac. hipúrico & N.D. & N.D. \\
Ac. hidroxihipúrico & $1,25 \pm 0,05$ & $0,17 \pm 0,04$ \\
Ac. 3-fenilpropiónico & N.D. & N.D. \\
Ac. 3-(4-hidroxifenil)propiónico & $2,82 \pm 0,05$ & $0,08 \pm 0,01$ \\
\hline N.D. $=$ no detectable & &
\end{tabular}


Tabla 5. Actividad antioxidante (TEAC) de mezclas equimolar de ácidos

\begin{tabular}{ccc}
\hline Combinación de ácidos & ABTS (mM) & FRAP (mM) \\
\hline Ac. gálico /Ac. siríngico & $3,44 \pm 0,06$ & $3,32 \pm 0,14$ \\
Ac. gálico /Ac. protocatéquico & $5,97 \pm 0,18$ & $4,71 \pm 0,23$ \\
Ac. gálico /Ac. 3, 4-dihidroxifenilacético & $5,46 \pm 0,06$ & $3,32 \pm 0,13$ \\
Ac. gálico /Ac. caféico & $4,21 \pm 0,16$ & $7,57 \pm 0,33$ \\
Ac. gálico /Ac. cumárico & $4,57 \pm 0,17$ & $2,61 \pm 0,11$ \\
Ac. gálico /Ac. 3,4-dihidroxifenilacético & $6,20 \pm 0,36$ & $10,84 \pm 0,90$ \\
/caféico & & \\
Ac. gálico /Ac. 3, 4-dihidroxifenilacético & $6,42 \pm 0,16$ & $8,12 \pm 0,23$ \\
/Ac. cumarico & $3,09 \pm 0,05$ & $3,05 \pm 0,21$ \\
Ac. siríngico /Ac. protocatéquico & $2,29 \pm 0,20$ & $1,90 \pm 0,11$ \\
Ac. caféico /Ac. ferúlico & $2,52 \pm 0,23$ & $1,22 \pm 0,05$ \\
Ac. caféico /Ac. cumárico & $3,42 \pm 0,30$ & $2,67 \pm 0,27$ \\
Ac. caféico /Ac. 3,4-dihidroxifenilacético & $2,43 \pm 0,04$ & $0,61 \pm 0,04$ \\
Ac. cumárico /Ac. ferúlico & $3,70 \pm 0,25$ & $4,57 \pm 0,04$ \\
Ac. cumárico/Ac. 3, 4-dihidroxifenilacético & & \\
Ac. 4-hidroxi-3-metoxifenilacético /Ac. & $5,31 \pm 0,16$ & $5,41 \pm 0,02$ \\
3,4-dihidroxifenilacético & & \\
Ac. 4-hidroxi-3-metoxifenilacético /Ac. & $3,42 \pm 0,30$ & $2,31 \pm 0,56$ \\
\hline & & \\
\hline
\end{tabular}

También se analizó otros compuestos derivados de flavonoides por la microbiota de intestino humano de estructuras diversas, se puede observar que sólo presenta capacidad antioxidante por el método ABTS*, lo que nos permite sugerir que de acuerdo a su estructura sólo presenta la capacidad de donación de H (tabla 4).

Al efectuar una evaluación de las posibles interacciones de las mezclas de los ácidos fenólicos por ambos métodos, se observa un efecto predominantemente antagónico con respecto a la suma individual de sus actividades, no existiendo al parecer un posible patrón estructural que explique dicho comportamiento; lo que sugiere que debe formarse complejos intramoleculares estables entre ellos que atenúan su actividad antioxidante en las condiciones del experimento. Estos complejos intramoleculares podrían darse debido a enlaces de hidrógeno entre los grupos carbonil y grupos ortodihidroxil. Sólo en el caso de la mezcla de ácido siríngico ácido protocatéquico (3,4-dihidroxibenzoico) presenta un efecto sinérgico. 
Es probable que algunos otros fenómenos estén involucrados como la polaridad de las moléculas, así como la proporción o la cinética de reacciones de los antioxidantes en el medio de reacción.

\section{CONCLUSIONES}

- Los métodos ABTS y FRAP miden diferentes aspectos del comportamiento de las sustancias, lo que determina su diferente perfil antioxidante.

- Los ácidos fenilacéticos presentan mayores valores con el FRAP, los ácidos hidroxicinámicos con el ABTS, mientras que los ácidos hidroxibenzoicos es variado.

- Por el método FRAP los ácidos fenólicos más activos resultaron aquellos compuestos dihidroxilicos en posición meta y para con respecto al grupo carboxílico, lo que no se puede establecer en el ABTS ya que esto varía en los tres tipos de ácidos fenólicos estudiados.

- Se confirma que la actividad antioxidante depende tanto de la posición como del número de grupos $\mathrm{OH}$ en el anillo.

- El efecto de las interacciones de la mezclas de los ácidos fenólicos es predominantemente antagónico con ambos métodos.

\section{AGRADECIMIENTO}

Al grupo de Investigación de Polifenoles de la Universidad de Salamanca, España.

\section{REFERENCIAS BIBLIOGRÁFICAS}

1. 1. Aura AM, O'Leary KA, Willianson G, Ojala M, Bailey M, Puupponen-Pimia R. Quercetin derivatives are deconjugayed and converted to hydroxyphenylacetic acids but not methylated by Human Fecal flora in vitro. J Agri Food Chem. 2002; 50: 17225-30.

2. Déprez S, Brezillon C, Rabot S, Philippe C, Mila I, Lapierre C, et al. Polymeric proanthocyanidins are catabolized by human colonic microflora into low-molecularweight phenolic acids. J Nutr. 2000; 130: 2733-2738.

3. Lambert JD, Sang S, Yang CS. Biotransformation of Green Tea Polyphenols and the Biological Activities of those Metabolites. Mol Pharm. 2007; 4(6):819-25.

4. Prior RL,Wu X, Schaich K. Standardized methods for the determination of antioxidant capacity and phenolic in foods and dietary supplements. J Agric Food Chem. 2005; 53: 4290-4302.

5. Hollman PC, Katan MB. Absorption, Metabolism and health of dietary flavonoids in man. Biomed Pharmacother. 1997; 51(8): 305-310.

6. Al-Farsi M, Alasalvar C, Morris A, Baron M, Shahidi F. Comparison of antioxidant activity anthocyanins, carotenoids, and phenolic of three native fresh and sun-dried date 
(Phoenix dactylifera) varieties grown in Oman. J Agric Food Chem. 2005; 53: 75927599.

7. Huang D, Ou B, Prior RL. The Chemistry behind Antioxidant Capacity Assays. J Agric Food Chem. 2005; 53: 1841-1856.

8. Rice-Evans C, Miller N, Paganga G. Structure-antioxidant activity relationship of flavonoids and Phenolic acid. Free Radic Biol Med. 1996; 20 (7): 933-956.

9. Lee HC, Jenner AM, Low CS, Lee YK. Effect of tea phenolics and their aromatic fecal bacterial metabolites on intestinal microbiota. Res Microbiol. 2006; 157(9):876-84.

10. Benzie I, Strain J. Ferric reducing/antioxidant power assay: direct measure of total antioxidant activity of biological fluids and modified version for simultaneous measurement of total antioxidant power and ascorbic acid concentration. Methods Enzymol. 1999; 299: 15-27.

11. Re R, Pellegrini N, Proteggente A, Pannala A, Yang M, Rice-Evans C. Antioxidant activity applying an improved ABTS radical cation decolorization assay. Free Radic Biol Med. 1999; 26(9-10):1231-7.

12. Frankel E, Meyer A. The problems of using one-dimensional methods to evaluate multifunctional food and biological antioxidants. J Sci Food Agric. 2000; 80:1925-41.

13. Chen JW, Zhu ZQ, Hu TX, Zhu DY. Structure-activity relationship of natural flavonoids in hydroxyl radical-scavenging effects. Acta Pharmacol Sin. 2002; 23(7): 667-72. 